\title{
Applying Genetic Algorithm to Conceptual Clustering
}

\author{
Non-member Myung-Mook Han (Osaka City University) \\ Non-member Shoji Tatsumi (Osaka City University) \\ Member Takaaki Okumoto (Osaka Institute of Technology)
}

\begin{abstract}
In this paper, we propose the use of Genetic Algorithm (GA) as a key element in the design of robust learning system. We describe a GA-based concept learner called GAIL, capable of inducing descriptive concepts in conjunctive statements. A motivation to use a GA is that GAs are able to explore large domains because they make use of a multipoint search strategy and are suitable to exploit massive parallelism. By incorporating a GA with a traditional method, the system has several important features. First, the system is robust. Second, the system introduces the modified evaluation criteria to evaluate the quality of a clustering. Finally, the system searches for an individual which refers to all the concepts at the same time. The effectiveness of this approach is illustrated by two exemplary problems and the clusters obtained with this system correspond well to categorization satisfied by people.
\end{abstract}

Key words: Genetic Algorithm, Conceptual Clustering, Machine Learning

\section{Introduction}

Machine learning may be defined as any process which envolves designing computer programs to build new knowledge or to improve already possessed knowledge. One of the basic types of learning is inductive learning which learns any concepts or ideas by generalizing specific facts. It has received considerable attention in artificial intelligence. An important goal of inductive learning is to create a meaningful classification of observed objects or events.

Classification process is regarded as the first step in developing a theory about a collection of observations. This process is a form of learning from observation, and its purpose is to organize given observations into a hierarchy of meaningful categories.

Unitil now, the most of the past works on this field have been done under the title of numerical taxonomy. These methods are based on the application of a mathematical measure of similarity between objects. Each class of objects is taken as the collection of the objects whose intraclass similarity is high, and interclass similarity is low. However, such approaches have significant limitation because they do not take into consideration any background knowledge among the object attributes or the global concepts that could be used for characterizing the object configurations. As a result, classification obtained by the traditional method is often difficult to interpret conceptually.

To overcome this, conceptual clustering was introduced by Michalski and Stepp ${ }^{(1) \sim(3)}$ as an extension of processes of numerical taxonomy. The main advantage of the conceptual clustering is the ability to capture the properties of object clusters that characterize a cluster as a whole and are not derivable from properties of individual entities. To explain this idea, let us consider the example shown in Fig. 1, from reference ${ }^{(4)}$.

A person considering this figure would typically regard the observed points as representing two circles. Thus, the point $\mathrm{A}$ and $\mathrm{B}$, although closer to each other than to other points, are placed into different clusters. CLUSTER/ 2 has been proposed as a conceptual cluster. ing algorithm to make such classifications.

The algorithm performs by alternately choosing a set of seed objects (one per class) and using the seeds to lead inductive inference to produce mutually disjoint descriptions of object classes. Each cluster description must cover the given seed but no other seeds, and different seeds are used over many iterations until it optimizes the clustering quality criterion. But the above method for determining an optimized clustering from seed requires NP-complete algorithms. Therefore, if the problem size becomes larger, the greater computaional complexity the more disadvantageous the method. Also, 


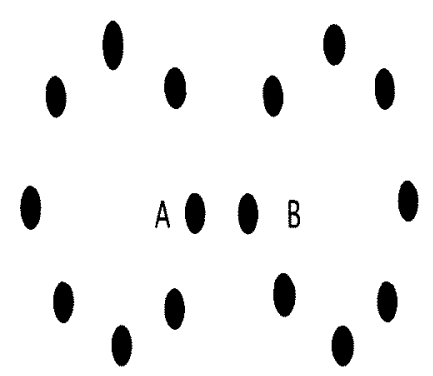

Fig. 1. An illustration of conceptual clustering (referred from reference ${ }^{(4)}$ ).

because CLUSTER/ 2 handles one concept at a time, the computational cost is relevant. Finally, CLUSTER/2 introduces the Lexicographical Evaluation Functional (LEF) to evaluate the quality of clustering. But there is no criteria for choosing one or another of the various modification. To solve the above problems, the parallel processing and the stochastic algorithm have been introduced in order to obtain the approximate optimal solution. In this paper, we propose the GAIL (Genetic Algorithm-based Inductive Learning) system which adapts GA as a key element and has the parallel characteristic. GA is a parallel, global search technique modeled with the Darwinian principle of survival and reproduction of the fittest ${ }^{(5)(6)}$. GA is a repeated process which maintains a set of strings, called the population. During each repeated step, the individuals in the current population are evaluated by the fitness function and as its base, the next population is formed through the application of genetic operators. In GAIL, GA is presented for conceptual clustering that is based on a problem-specific representation method and problemspecific genetic operators. We will introduce the modified evaluation criteria to evaluate the quality of a clustering. We will use artificial data and implement two experiments which are evaluated by the modified evaluation criteria. As a result, we obtained the meaningful concepts of the events.

In Sect. 2, we will explain the conceptual clustering and its relation to the GAs. Sect. 3 introduces GAIL system which provides a framework for combining $G A$ with CLUSTER/2 method, and GAIL is tested on two exemplary problems in Sect. 4. Finally, we conclude the paper in the Section 5.

\section{Conceptual Clustering}

Clustering is a process of grouping given set of objects into meaningful classes. A number of clustering algorithms have been proposed, each utilizing its own notion of similarity and concept of cluster ${ }^{(7)-(9)}$. These algor. ithms can be broadly classified into the two types ${ }^{(10)}$. One is the type in which knowledge is implicitly expres. sed. The Single-Linkage algorithm and the K-means algorithm are included in this type ${ }^{(7)}$. Another is the type in which knowledge is explicitly expressed. Example of such an algorithm includes the Conceptual Clustering algorithm ${ }^{(1)}$. A specific form of the conceptual clustering is the CLUSTER/2 in which classes are described by the conjunctive statements.

\subsection{Outline of CLUSTER/2}

This section briefly describes the conceptual clustering method of CLUSTER/2. The main idea behind CLUSTER $/ 2$ is that objects are classified into groups that are concisely described by conjunctive statements and descriptive concepts of the groups are optimized according to a global criterion of classification quality. The problem posed in the structure of CLUSTER/2 is defined as follows:

Given

- A set of objects,

- A set of attributes to describe the character of object, and

- The background knowledge (problem constraints, properties of attributes, and a criterion for evaluat. ing the quality of candidate classifications); and find

- A hierarchy of object classes, and their description in the form of conjunctive statements which maxi. mize a clustering preference criterion.

CLUSTER/2 method is applied to an example that can be expressed as the list of variables consisting of nominal, linear, and structured one. A nominal variable is one whose values form a set with unordered structure; for example the set of colors. A linear variable is one whose values are linearly ordered; for example weight. A structured variable is one whose values are ordered hierarchically. For example, the shape might be hierarchical, having subsets such as pentagon and polygon.

The way to measure a clustering preference criterion in CLUSTER/2 is to define various elementary criteria and to combine them into one global criterion, called the Lexicographical Evaluation Functional (LEF). Examples of elementary criteria are the fit between the clus. tering and the events, the simplicity, the commonality, the disjointness, and the discrimination index. The LEF is composed of a sequence of elementary criteria, wherein the increase of value improves the quality of the clustering. First, all clusterings are evaluated on the 
first elementary criterion. Those that score best or within the given range from the best are maintained. Those maintained are then evaluated on the second elemontary criterion. This process continues until either the best clustering remains or the list of elementary criteria is emptied. In the latter case, the algorithm selects one arbitrarily. The algorithm composes of two phases: a clustering phase and a hierarchy-building phase. The clustering phase divides events into classes, so that the resultant clustering maximizes the given criterion of clustering quality LFE. The hierarchy. building phase uses the clusering phase to decide a hierarchy of clusters.

The basic algorithm of a clustering phase is as follows:

The clustering phase algorithm begins by alternately selecting a set of seed objects, one per class (STEP 1). Next a star is constructed for each seed object against the other seed objects (STEP 2). And by appropriately modifying and selecting complexes from stars, an optimized clustering that optimizes the clustering quality criterion LEF is built (STEP 3). If the termination criterion is satisfied, the algorithm terminates (STEP 4). Otherwise, new seeds are chosen from sets of observed events contained in complexes of the generat. ed clustering, one seed per complex (STEP 5). After selecting seeds, the algorithm begins from STEP 2 again.

The use of terms seed, star, and complex is originated from Michalski and Stepp ${ }^{(1)}$.

\section{2 Conceptual Clustering method by GA}

One of the most important characteristics of GAs is the domain independence of its search mechanism. This is the origin of the many successes of GAs and of many restrictions in other applications. Such arguments are similar to those demanding more task-specific AI method 20 years ago. At first, general problem solvers were invented. It was soon revealed that it was necessary to incorporate problem-specific knowledge. Since many opportunities exist for incorporating problemspecific knowledge into GAs, it was regarded as a method for improvement in many domains. This paper explores one of these opportunities in the context of the conceptual clustering problem of CLUSTER/2. CLUS$\mathrm{TER} / 2$ has the potential to be a useful method in analyzing data. But the construction of an optimized clustering that optimizes the clustering criterion LEF is the most computationally costly step of the method (STEP 3 of the clustering phase). Because the stars may hold many complexes, the above step is extremely inefficient for solving real-world problems. Therefore, if the number of events and attributes is large, it is faced with a significantly greater computational complexity. $T_{0}$ avoid the combinatorial explosion, the parallel processing and the stochastic algorithm have been introduced as a way of obtaining the approximate optimal solution. GA, a probabilistic search algorithm for the optimal solution, is used in this paper. In the clustering method by $\mathrm{GA}$, we search the desirable clustering through the combination of classifying attribute. That is, the individuals represent the scope of classifying attribute for each class and then the generation repeats the variation of scope to determine the individual for which the most desirable clustering is obtained based on the evaluation criteria.

\section{GAIL: Genetic Algorithm-based Inductive Learning}

We are now going to describe GAIL, our GA-based inductive concept learner. At the center of the system is a GA for searching the attribute-based space. Fig. 2 shows a pseudo-code of the GA used. $P(t)$ represents a population of individuals at time $t$.

We shall describe an outline of the GAIL. The steps are:

(1) Initial population are generated.

Using the heuristic knowledge, we construct the initial population in order of nominal, linear, and structured variables.

(2) Evaluate each individual in population.

The population of individuals are evaluated by the LEF. If the GAIL is implemented on a massively parallel computer, each individual is assigned to a processor and is evaluated simultaneously ${ }^{(12)}$.

(3) A termination criterion is examined.

The algorithm terminates when a specified number of generations is reached.

(4) An optimized clustering is built by the genetic operators.

The combination of the scope of classifying attribute

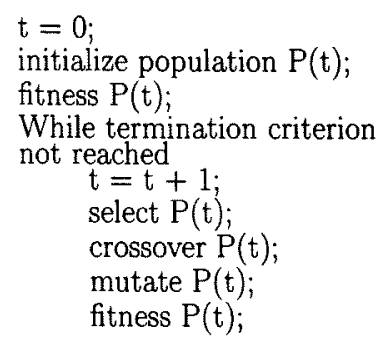

Fig. 2. The GA in GAIL. 
Given :

E - a set of data events

$K$ - the number of clusters

LEF - the clustering quality criterion

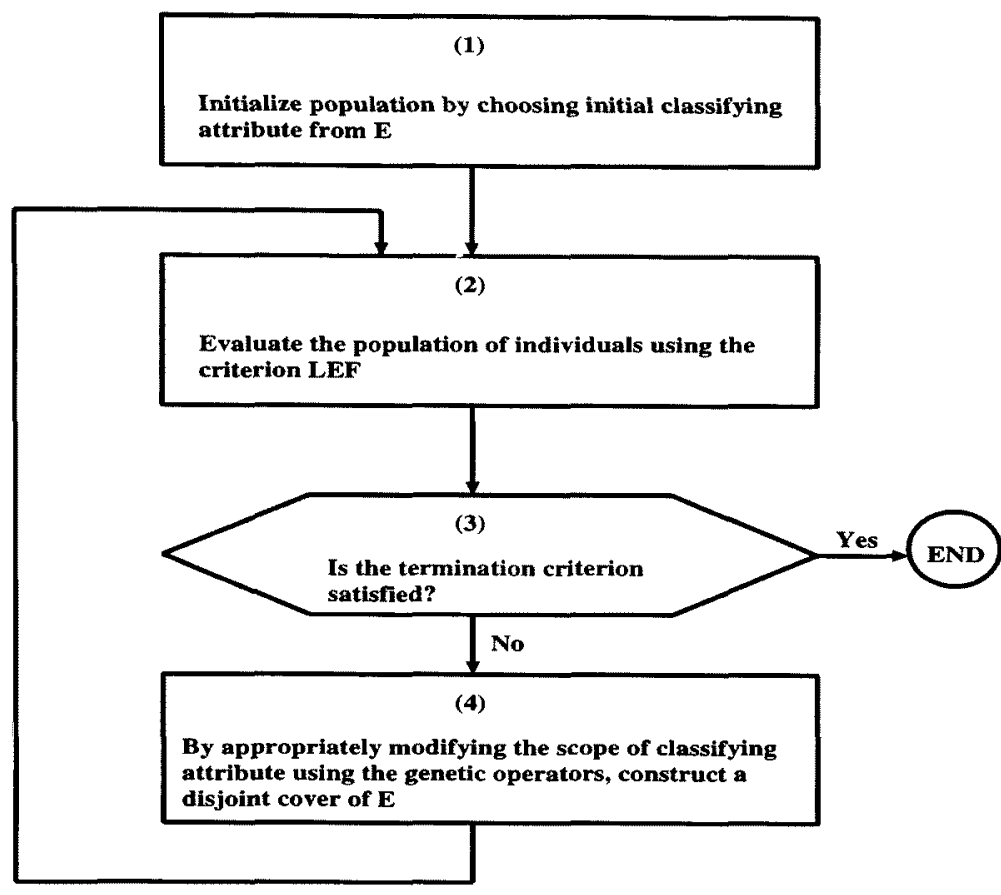

Fig. 3. The flow chart of the GAIL.

is tested to see whether it contains interesecting scope. If so, the scope is made disjoint. Also, if the GAIL is implemented on a massively parallel computer, it will advantage a computational speed.

After constructing a disjoint cover, a new iteration of the algorithm begins from step 2. The GAIL is summarized by the flow chart in Fig. 3.

The most important issues and approaches used in GAIL are as follows :

\section{1 Encoding}

To represent the optimization problem's variables, the encoding method is essential to the GA. In this section we will describe the encoding method for GAIL system. Before we do that, we need to define several terminologies to improve the understanding as follows:

〈Definition 1〉 Clusters which is described by single concepts are characterized by logical products of relations on selected object attributes (conjunctive concepts).

〈Definition 2〉 The event space is the description space which is spanned by variables $x_{1}, x_{2}, \cdots, x_{n}$. Each point (event) in the event space is represented by a vector of specific values of variables.
〈Definition 3〉 Given a collection of objects $E$, each gene-factor divides the collection into certain subsets. Let $E_{1}, E_{2}, \cdots, E_{k}$ be such subsets of $E$, and let $d_{i}$ denote a description of subset $E_{i}$. An union of a set of subsets is the set $E$, and a descriptions of a set of subsets are all disjoint. The gene-factor uses conjunctive forms with internal disjunction and each individual consists of genefactors.

The encoding method relies on the character of the problem variables. Even though there are several types of the encoding method, the binary method that each individual in the population consists of a string of binary digits is used. In this paper, the gene-factor is regarded as the number of sections in the individual. Each genefactor holds 16 columns and the individual is made up three gene-factors maximumly. The first element of an individual represents the number of gene-factors. Generally speaking, if the number of gene-factors is $k$ and the length of a gene-factor is $l$, the length of individual becomes $k \times l+1$.

We explain the encoding method according to the variables.

- Nominal variable: It describes the class number 
$12345678910111213 \cdots 49$

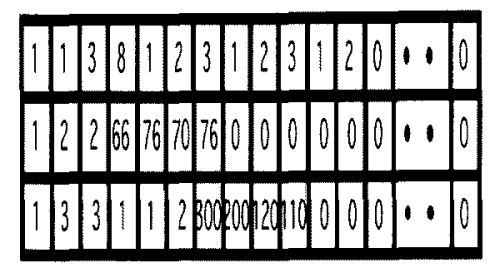

Fig. 4. An example of the encoding method.

that the nominal variable belongs to.

- Linear variable: The linear variable is represented by both the scope of each class's value and the threshold value to be used for the partitioning.

- Structured variable: We limit its organization to a tree structure and mention through the block of tree beleoning to each class. The edges from each node are numbered in order and the structured variable is represented by their number of the edges from the root.

For example, suppose that there is a population of 3 individuals illustrated in Fig. 4.

Each individual is consists of 49 columns. Also, the first (second, third) individual is constructed by the nominal (linear, structured) variable. In the first individual, the first column shows there is only one genefactor. The second column indicates that the nominal variable is the first attribute of a given data and the third column expresses 3 classes to be used. The fourth column exhibits the number of attribute value is eight, and each value is represented by the class number from the 5 th column to the 12 th column. In the second individual, the first, second, and third column express the same as in the first individual. The 4 th and the 5 th column indicate the lower bound and the upper bound of variable. The 6 th column shows the partitioning threshold value is 70 and the 7 th column is the upper bound of variable. Finally, in the third individual, the first, sec. ond, and third column indicate the same as the first and second individual. The 4 th (5th) column indicates that the contents of the 7 th ( 8 th) column belong to the 1 class and the 6 th column exhibits the contents of the 9 th. The 10th column belongs to the 2 class.

\section{2 Initial population}

The initial population must contain many individuals, each of which is a potential solution. This process might be random, or it might incorporate the task-heuristic knowledge. We created the initial population using the heuristic knowledge. The first (second, third) individual was constructed by the nominal (linear, structured)
Table 1. The sparseness of each attribute.

\begin{tabular}{|l|l|}
\hline $\begin{array}{l}\text { Attribute } \\
\text { Value }\end{array}$ & The formula of the sparseness \\
\hline \hline $\begin{array}{l}\text { Nominal } \\
\text { Variable }\end{array}$ & $\begin{array}{l}\text { the number of names to perform after the partition }] \\
/ \text { [the whole number of names] } \times 100\end{array}$ \\
\hline $\begin{array}{l}\text { Linear } \\
\text { Variable }\end{array}$ & $\begin{array}{l}\text { [the range of value to perform after the partition] } \\
/[\text { the whole range of values] } \times 100\end{array}$ \\
\hline $\begin{array}{l}\text { Structured } \\
\text { Variable }\end{array}$ & $\begin{array}{l}\text { (1-([the number of event to be integrated structually] }-1) \\
/[\text { the whole structual number of events] }) \times 100\end{array}$ \\
\hline
\end{tabular}

variable, and it repeated in order of variable.

\section{3 Fitness Function}

In addition to the above issues, it is important to choose a fitness function. We selected a fitness function which consists of three criteria, the sparseness, the simplicity, and the scattering. The sparseness and the simplicity are picked from CLUSTER/2 method.

The sparseness of event is considered as the measure to judge whether the cluster description is suitable for describing the actual data. Suppose that the complex including observed and unobserved events to be classified is $\delta$. Here complex is used as algebraic topol ogy meaning ${ }^{(1)}$. If the whole number of exents is $t(\delta)$, and if the number of unobserved events is $s(\delta)$, the sparseness can be represented as $r(\delta)=s(\delta) / t(\delta)$. The high sparseness means that the number of the unobserved events is large within the cluster and that the cluster description is not identical with the actual data. When the number of attribute is $n$, the sparseness over any partition of cluster is as follows.

The sparseness $=\left(\sum^{n}\right.$ [the sparseness of each attribute for the partition] ) $/ n$.

According to the attribute, the formula of the sparse. ness are shown in Table 1.

Also, the simplicity of cluster description can represent how simple the cluster description is.

The simplicity of cluster $=(1-$ the necessary number of attribute for cluster partition] / [the whole number of attributes] ) $\times 100$.

The scattering of events means that there is little difference among the clusters in number of events. If the number of partition is $k$, the scattering of events is as follows.

The scattering of events $=\left(1-\sum_{i=1}^{k} \mid \quad\right.$ the number of events of the ith cluster] - ( [ the whole number of events] $/ k) \mid) /(k \times$ [the whole number of events] $) / 2(k$ $-1)) \times 100$.

So the evaluation criteria is proposed as folows. The fitness function $=\alpha \times(100-$ the sparseness $)+\beta \times$ (the simplicity of cluster) $+\gamma \times$ (the scattering of 
event).

Here, $\alpha, \beta, \gamma$ are the parameters of the evaluation criteria and the above evaluation function is an experimental choice rater than a theoretical one.

\section{4 Genetic operators}

Genetic operators change individuals within a population to produce new individuals. Among the genetic operators, crossover and mutation are used.

We specify the genetic operators in GAIL system using the following definition and explain them with a simple example.

〈Definition 4> Genetic operators produce new (possibly better) individuals by making an alternative to the current conjunctive forms. Crossover transforms individuals to new states in the event space by changing the gene-factors between parent individuals. Since it is so complicated to change the genefactors, a certain rule has to be decided to perform the crossover. Mutation transforms individual to new state in the event space by changing a conjunctive form within the gene-factor.

The following simple example illustrates some further details of the genetic operators. There are eight objects, each described by two variables: Attribute $A$ and Attribute B. Object descriptions are presented in Table 2. For simplicity, let us assume that the goal is to partition objects into only two classes $(k=2)$ using a fitness function.

Table 2. The example of clustering.

\begin{tabular}{ccc}
\hline Event & Attribute A & Attribute B \\
\hline$e_{1}$ & $\mathrm{a}$ & 1 \\
$e_{2}$ & $\mathrm{a}$ & 8 \\
$e_{3}$ & $\mathrm{~b}$ & 5 \\
$e_{4}$ & $\mathrm{~b}$ & 10 \\
$e_{5}$ & $\mathrm{c}$ & 1 \\
$e_{6}$ & $\mathrm{c}$ & 9 \\
$e_{7}$ & $\mathrm{~d}$ & 5 \\
$e_{8}$ & $\mathrm{~d}$ & 3 \\
\hline
\end{tabular}

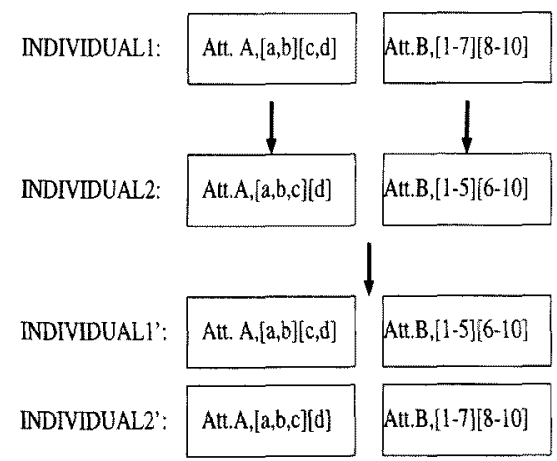

Fig. 5. The crossover of individual.
Next, we explain the method of genetic operators. Suppose that there are two individuals, as shown in Fig. 5.

Individual 1 holds the information that the classes are divided into the group (a, b) and the group (c, d) using the attribute $A$, and divided into the group (1-7) and the group (8-10) using the attribute $B$

With the same method, individual 2 holds the information that the classes are divided into the group $(\mathrm{a}, \mathrm{b}, \mathrm{c})$ and the group (d) using the attribute $\mathrm{A}$ and divided into the group (1-5) and the group (6-10) using the attribute $B$. With the crossover to exchange the information

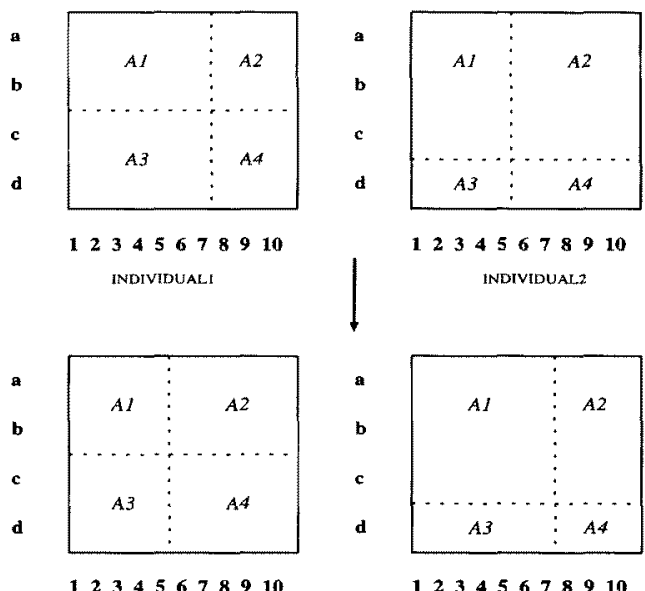

INDIVIDUALL!

INDIVIDUAL2:

Fig. 6. The event space of crossover

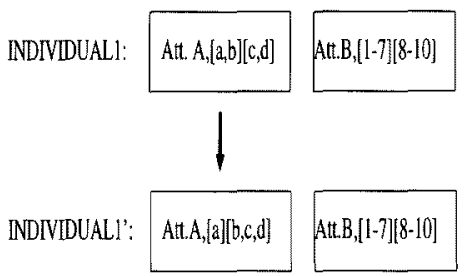

Fig. 7. The mutation of individual.

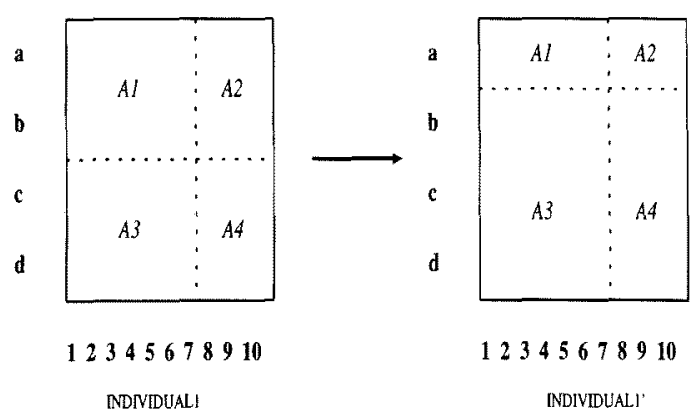

Fig. 8. The event space of mutation. 
(Fig. 5), it would be the group (a, b) and the group (c, d) using the attribute $A$, and the group (1-5) plus the group (6-10) using the attribute B in the individual 1'. Also, it would be the group (a, b, c) and the group (d) using the attribute $A$. The group (1-7) and the group (8 $-10)$ are using the attribute $B$ in the individual 2'. Fig. 6 shows a geometrical representation in the event space of crossover.

With the mutation (Fig. 7), the information within the unit is changed randomly. Fig. 8 illustrates a geometrical representation in the event space of mutation. After new individuals are produced by genetic operators, GAIL selects two classes among four categoris ( $A 1, A 2$, $A 3$, and $A 4$ ).

\section{Experiments}

We first will describe the outline of experiment. The initial population consists of the individuals which hold one gene-factor. That is, each individual is constructed by one attribute variable and partitioned into two or three classes on the basis of the variable types. Nominal variable separates a collection of events through the given class number. Linear variable separates a collection of events through the scope of each class's value. And structured variable divides a collection of events into the block of tree belonging to each class.

The fitness value of an individual is calculated by the fitness function which is made up the sparseness, the simplicity, and the scattering. To calculate the sparseness, we firstly investigate which classes include the events based on the classifying attribute. Then we divide classes according to the number of gene-factors. Finally, the sparseness is calculated by adding up the sparseness of each attribute for the divided classes.

Once all individuals in the population have been evaluated, their fitnesses are used for selection. Selection is implemented by keeping a high-fitness individual as a parent to perform the crossover operator. Genetic operators such as crossover and mutation are applied to the selected individuals to produce a new population of individuals.

Crossover operator creates new individuals by combining genes of individuals to be a parent. This operator is the most important one among the genetic operators in the GAs. We combine the properties of individuals through the sum of gene-factors in parents. That is, if the number is 2 or 5 , it produces individuals to hold two gene-factors. If the number is 3 or 6 , it creates individuals of two or three gene-factors. Finally, if the number is 4 , the individuals of one gene-factor are created.
Since crossover operator relocates the position of genefactor in an individual, it processes these individuals to produce new individuals which may have several genefactors. Also, mutation operator changes the par titioned structure of gene-factors in an individual. By transforming the previous set of good individuals to a new one, the genetic operators generate a new set of individuals that have a better than the average fitness. When this cycle is repeated for many generations, the overall fitness of the population generally improves, and the individuals in the population represent improved clusters which is satisfactory for people. The described method was applied to two exemplary problems, the first dealing with classifying a microcomputer, and the second dealing with constructing a classification of the vertebrate animal.

\section{1 Experiment 1}

To compare with CLUSTER/2 method, we used the problem which had been solved by CLUSTER/2 $\operatorname{method}^{(2)}$. The problem is to develop a meaningful classification of popular microcomputers and each microcomputer is described in terms of the variables.

The number of events is 12 and the number of variable is 5. "RAM", "ROM" and "Keys" are linear variables. "MP" and "Display type" are structured variables with domains.

The program was given the above data and was told to use the different parameter settings of the evaluation criteria. We had implemented the experiment with the following parameters.

1. $\alpha=1, \beta=0, \gamma=0$

2. $\alpha=0, \beta=0, \gamma=1$

3. $\alpha=1, \beta=1, \gamma=1$

The results of this experiment are shown in Fig. 9.

The case 1 shows the result when evaluated by the sparseness only. The evaluation of the sparseness makes an identical degree between clustering and events larger, but if only this criteria has been used, it is inclined to implement the clustering which separates largely different events from events themselves. Fig. 9 (a) represents the result distinctively. At the first level in Fig. 9 (a), GAIL split the microcomputers into two clusters according to the type of Keys. One cluster is composed of keys' number on keyboard which is 92 . The other cluster has the microcomputers utilizing a keys' number on keyboard that is less than 92 . In the second level of clusters on the Keys $\neq 92$ branch of the hierarchy, there is no more partition according to three variables: Display, RAM and ROM.

The case 2 shows the result evaluated by the scatter- 
Applying GA to $\mathrm{CC}$

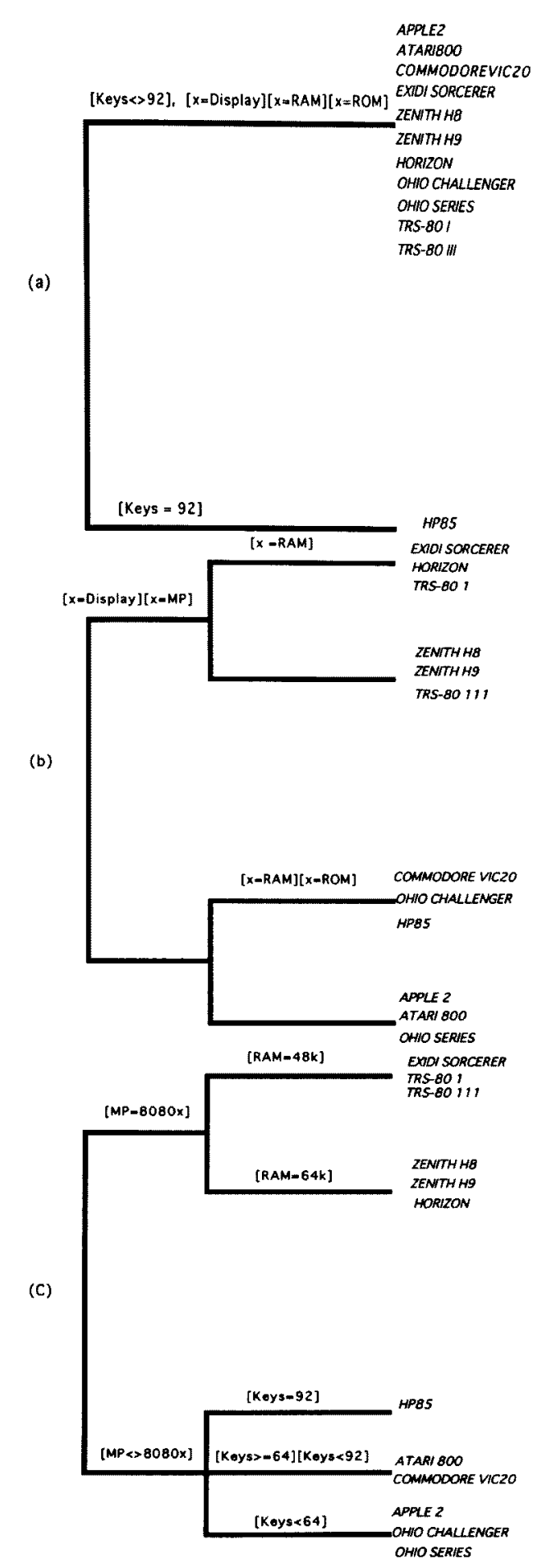

Fig. 9. Classification (1) of microcomputers generated by GAIL.

ing only. The evaluation of the scattering is inclined to implement the clustering which partitions the number of event in each cluster equally. The clustering hierarchy shown in Fig. 9(b) also contains two level. In the

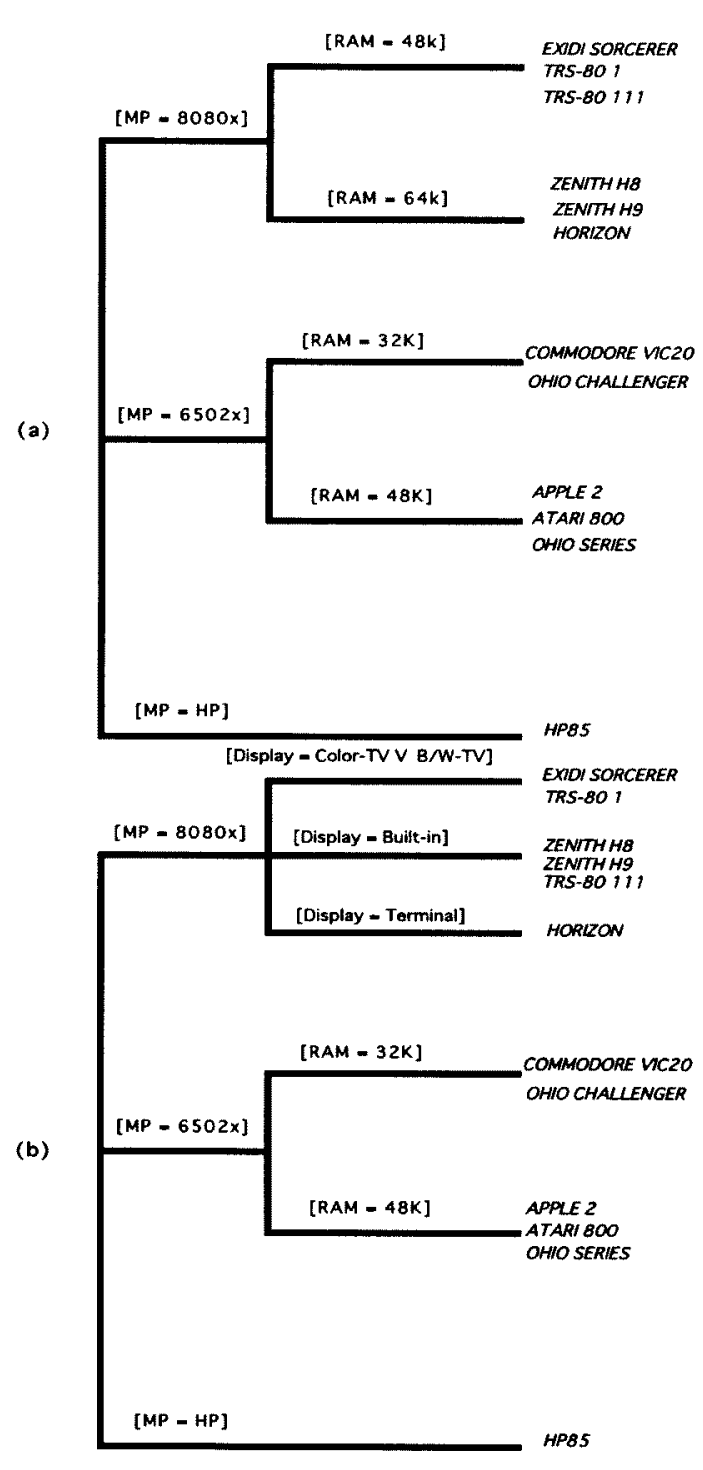

Fig. 10. Classification (2) of microcomputers generated by GAIL.

figure, 12 events are classified into 2 classes recursively and each class has 3 events.

The case 3 shows the result evaluated by the combination of evaluation criteria with the appropriate proportion, illustrated in Fig. 9(c).

We further investigated the optimized value of parameters. In the case $\alpha=2, \beta=1$, and $\gamma=1$, the clustering is also a two level hierarchy shown in Fig. 10(a).

In the case $\alpha=6, \beta=1$, and $\gamma=1$ shown in Fig. 10 (b), the structure was same as that of the case $\alpha=2, \beta=1$, and $\gamma=1$ except the $\mathrm{MP}=8080 \mathrm{x}$ cluster in level two. The $\mathrm{MP}=8080 \mathrm{x}$ cluster was partitioned into three clusters according to the Display type. Fig. 10 (b) presents the optimal clustering which is almost the same as the 


\section{2 Experiment 2}

The second problem is to classify vertebrates (animals having a spinal column). Thirty cases of vertebrate are given, and each characterized by the 11 multivalued variables.

The variables are included such as the skin shape of body, the number of heartchambers in a heart, the temperature of body, the fertilizable state, the parts of body which are used for moving, the way of eating, the outward shape, the inward shape, the reproduction period, a number of offsprings, and the environment in which animal acts.

Among them, the number of nominal variables is 8 , linear is 2 , and structured variable is 1 . These cases are drawn from 3 kinds of species. Ideally, a clustering method should partition these given cases into three groups corresponding to the variable. We also had implemented the experiment 2 with the following parameters.

1. $\alpha=1, \beta=0, \gamma=0$

2. $\alpha=0, \beta=0, \gamma=1$

3. $\alpha=1, \beta=1, \gamma=1$

The result of the case 1 which was evaluated by the sparseness only is shown in Fig. 11 (a).

At the first level, GAIL divided the vertebrates into two clusters according to three variables: Rperiod, Num and Ecology. In the second level of clusters which is no relation to water, there is no more partition. In the other cluster, GAIL split the vertebrate into two clusters according to three variabies: Body, Heart and Fert. The result of the case 2 to be evaluated by the scattering only is shown in Fig. 11(b). At the first level, GAIL separated the vertebrates into 2 groups according to two variables: Body and Ecology. In level two, the clusters are subdivided into three clusters such that each cluster contains the same number of events. The result of the case 3 to be evaluated by the combination of evaluation criteria with the appropriate proportion in shown in Fig. 11(c). At the first level in the hierarchy, GAIL split the vertebrate into 3 groups according to the type of body. In the second level of clusters on the Body=hair branch of the hierarchy, the vertebrate were separated into two clusters according to two variables: Ishape and Rperiod. In the other cluster, GAIL separated them into 2 groups according to the type of ecology and split accord. ing to two variables (Ishape and Ecology) in the third cluster. We examined the optimal value of parameters. In the case $\alpha=1, \beta=1$, and $\gamma=1$ at the first level and $\alpha$ $=6, \beta=1$, and $\gamma=1$ at second level, the simulation had

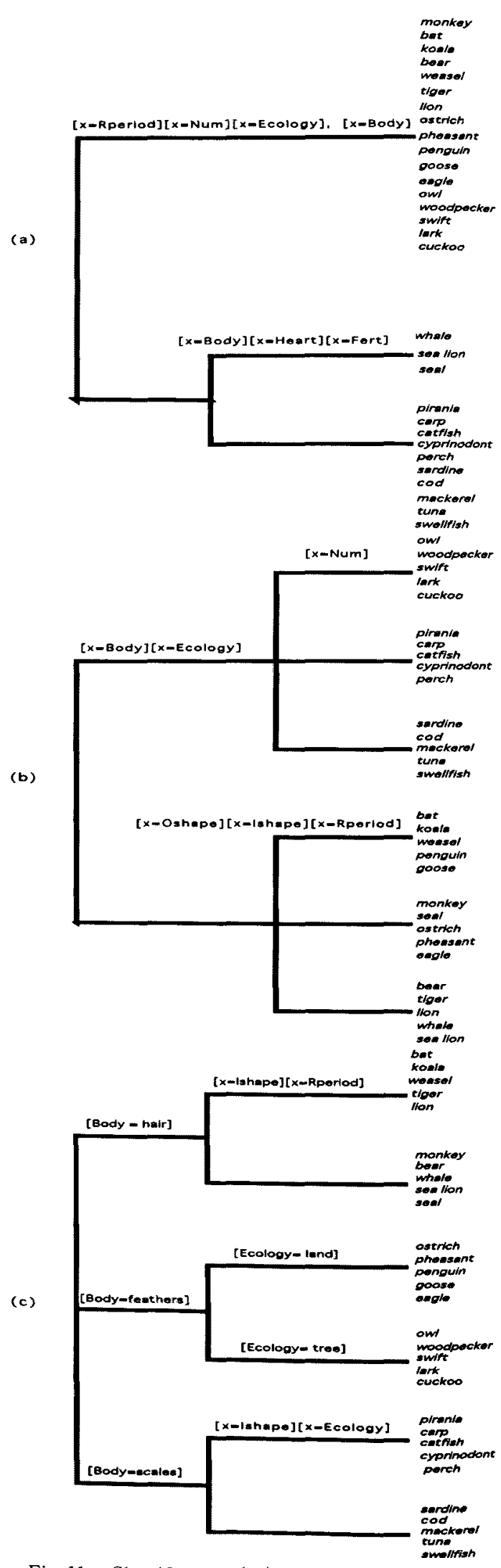

Fig. 11. Classification ( 1 ) of vertebrates generat. ed by GAIL. 


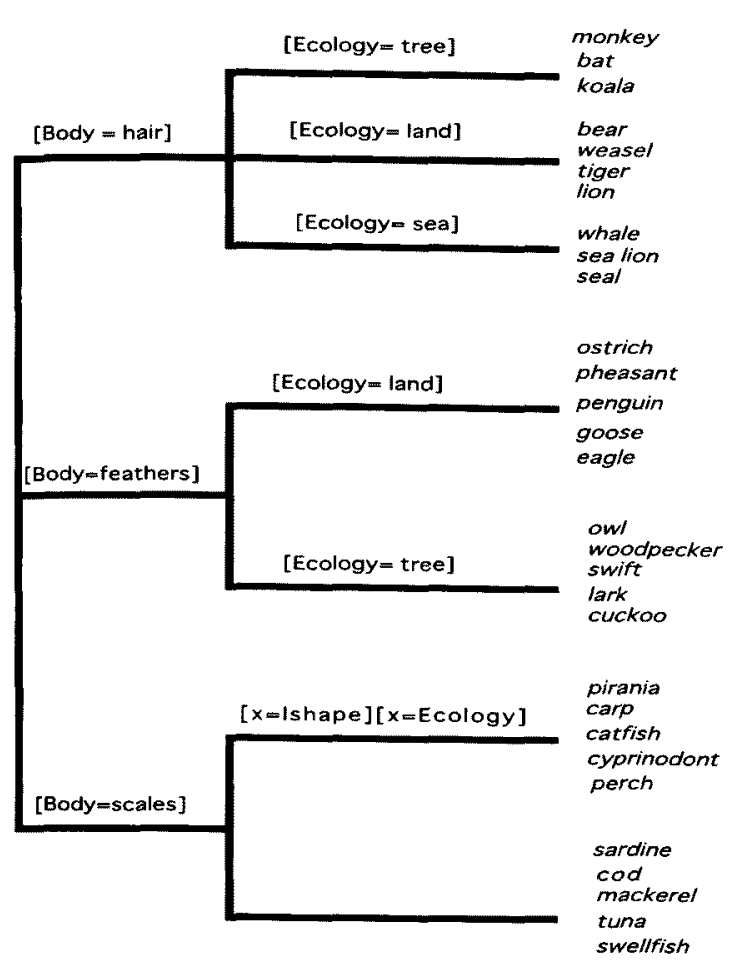

Fig. 12. Classification (2) of vertebrates generated by GAIL.

the optimal result which is in agreement with solutions satisfied by people, as shown in Fig. 12.

\section{3 Discussions}

Inductive learning algorithms employ bias to make learning more effective. In GAs, tasks are allocated to groups of genes based on their fitness. The GA's bias indicates that an exponentially increasing number of tasks will be allocated to sets of bits (schemata) appearing in the better performing individual. This bias is implemented by selection, crossover and mutation operators to supply specific biases that will affect the performance of the GA. Although GAs have been applied to a wide range of different problems in domain-independent style, the present paper introduces a knowledgeaugmented GA. The approach is based on the incorporation of domain-specific knowledge in the GA to use bias effectively. Therefore, this leads to a non-standard representation for chromosomes that contains all information related to the search performance and new domain-dependent crossover and mutation operators that utilize the additional information represented in the chromosomes. The typical domain-independent operators are no longer applicable because they would not produce consistent offspring. Knowlege-augmented crossover has been concentrated on the Traveling Sales. man Problem (TSP) ${ }^{(12)}$. Grefenstette proposed a heuristic crossover operator for the TSP. On the other hand, several authors developed the reordering operators that combine characters of inversion and crossover into a single crossover operator. In the experiment, we use a new crossover operator which should generate consistent offspring by combining attributes of two selected parent.

In Fig. 9(a), the fitness value converged to 73.6014 (78.0458) in $12(70)$ generations at the level 1(2). Also in Fig. 9(b), the maximum value 100 appeared in the 2 generations at the level 1 and in the 200(500) generations at the upper part (the lower part) of the level 2. It is noted that the best individual has usually two or three gene-factors in the above experiments. On the other hand, Fig. 9(c), 10(a), and 10(b) in which the simplicity of cluster was considered the maximum value appeared within 18 generations. During the implementa tion, the individuals of the early generations hold one gene-factor. When the process goes on, the individuals hold two or three gene-factors. Since the simplicity of cluster affects the individual in number of gene-factor, the experiments to consider the simplicity tend to converge in the early generations. In Fig. 11, the experiment (a) appeared the maximum value 96.9697 in the 30 generations at the level 1 and $98.4848(98.8636)$ in the 4 (4) generations at the upper part (the lower part) of the level 2. The experiment (b) presented the maximum value 100 in the 90 generations at the level 1 and $100(100)$ in the $200(30)$ generations at the upper part (the lower part) of the level 2. With the affect of the simplicity, Fig. 11(c) and Fig. 12 appeared the maximum values within 5 generations.

In the experiment, we have the optimal clustering in the case $\alpha=6, \beta=1$, and $\gamma=1$ and it is desirable to develope the method which decides the parameter values automatically in the near future. Also, since there is little difference between the integer and the real number of parameters in the fitness function, we have chosen the integer number in an understandable manner. The sparseness in the fitness function means the number of the unobserved events covered by a complex, and we can imagine that the higher the sparseness is, the larger it will be as a cluster for classifying given objects. Therefore, if we give priority to the sparseness by increasing the parameter $\alpha$ value, the clustering is fit for the events. We think that the sparseness relates to the number of events and attributes, but it is difficult to figure out the relation now.

From the experiments, we know that GAIL system is 
robust with the problem domain specific adaptations. Also, the system performs by integrating a set of simultaneous operations on a population of individuals, an essentially parallel operation, and it therefore natually lends itself to implementation in parallel hardware. Even when implemented on conventional computer, it converges on the optimal solution within reasonable speed.

\section{Conclusion}

We have described a new approach for using GAs as a key element in designing inductive learning system. Although GAs have been regarded as a viable method for search and machine learning problems, considerable research has been undertaken on improving GA performance. The paper used problem-specific parallel GA for improvement in machine learning domain.

CLUSTER/ 2 method forms an interesting technique, but there are considerable problems with the computational complexity and a criterion of clustering quality. To overcome the above problems, we have proposed the GAIL system which has the characteristic in the parallel $G A$.

In CLUSTER $/ 2$, the strategy for determining a clustering from seed is too inefficient and keeps from solving real world problems. When $n$ variables and $k$ seeds exist, a star may consist of $n^{k-1}$ complexes. Therefore, when $n=20$ and $k=3$, there are $n^{k-1}=400$ complexes, and the search tree has 400 -way branching at each node, and $400^{3}=64$ million leaves [1] . On the other hand, GAIL divides the event space into $k$. Therefore, when $n$ variables and $k$ the number of clusters exist, the event space becomes $k^{n}$, and GAIL divides $k^{n}$ into $k$. Also, GAIL system has the properties of parallel processing, and it will have the processing speed on the parallel computers. As a result, GAIL system is superior to CLUSTER/2 in view of computational point.

The proposed GAIL system has been applied to two exemplary problems and the clusters obtained with this system were satisfactory answers for people. Fisher's COBWEB is an incremental conceptual clustering system that carries out a hill-climbing search through a space of hierarchical classification schemes, and its results are dependent of input order. There fore, there are a number of limitations compared with GAIL system. Firstly, because of an inncremental system, the cost of incorporating an object into an existing classification tree may be two extreme. Secondly, COBWEB uses hill-climbing search strategy which are susceptible to becoming trapped in local optima.
Finally, objects in COBWEB are described in terms of only nominal attribute-value pairs. These prohibit COBWEB system from applying in sophisticated problem-solving domains.

The idea used here can be seen as a same case of ideas presented by Janikow ${ }^{(13)}$, where he described GIL (Genetic Induction Learning) exploring combinations of the traditional domain-independent GAs with some known problem-specific method. But the GIL system is quite complex and requires a number of paramters. Also, those ideas lie in learning production rules.

In conclusion, we believe that the proposd system would provide a quality alternative to existing methods and adds a new dimension to research in applying GAs to machine learning.

(Manuscript received August 26, 1996, rivised February 13, 1997)

\section{References}

(1) R.S. Michalski \& R. E. Stepp: "Learning from observation Conceptual clustering", In Machine Learning : An Artificial Intelligence Approach. Tioga, Palo Alto, CA. 331 363 (1983b)

(2) R. S. Michalski, \& R. E. Stepp: "Automated Construction of Classifications: Conceptual Clustering versus Numerical Taxonomy", IEEE Transactions on Pattern Analysis and Machine Intelligence, PAMI-5, No. 4, 396 410 July (1983a)

(3) R.E. Stepp \& R.S. Michalski: "Conceptual clustering: Inventing goaloriented classifications of structured objects", In Machine Learning: An Artificial Intelligence Approach. II, Morgan Kaufmann, San Mateo, CA. 471 498 (1986)

(4) J.H. Stephen: "Conceptual clustering and categorization: Bridging the Gap between Induction and Causal Models", In Machine Learning : An Artificial Intelligence Approach. in. Morgan Kaufmann, San Mateo, CA. 235 268 (1990)

(5) D. Lawrence, ed, : Genetic Algomithms and Simulated An nealing. Morgan Kaufmann Publishers, (1987)

(6) E. G. David: Genetic Algorithms in Search, Optimization and Machine Learning. Addison Wesley, (1989)

(7) M.R. Anderberg: Cluster Analysis for Applications. Aca. demic press, London, (1973)

(8) R.O. Duda \& P.E. Hart: Pattern Classification and Scene Analysis. Wiley, New York, (1973)

(9) A.K. Jain \& R. Dubes: Algorithms for Chustering Data. Prentice-Hall, Englewood Cliffs, NJ. (1988)

(10) V. Sridhar \& M. N. Murty: "A knowledge-based clustering algorithm", In Pattern Recognition Letters. North-Holland $511 \sim 517$ Sept. (1991)

(11) R.S. Michalski: "A theory and methodology of inductive learning", In Machine Learning: $A n$ Al Approach. Kaufmann, Los Altos, CA. (1983)

(12) M. M. Han, S. Tatsumi, Y. Kitamura, \& T. Okumoto: "Paral lel Genetic Algorithms based on a multiprocessor system FIN and its application", In IEICE Trans. Fundamentals, E78-A No. 11, 1595 1605, Nov. (1995)

(13) C. Janikow: Inductive Learning of Decision Rules in Attribute-Based Examples: a Knowledge-Intensive Geneti Algorithm Approach. Ph. D. dissertation, University of North Carolina at Chapel hill, July (1991) 
Myung-Mook Han (Non-member) He received the B.E. in

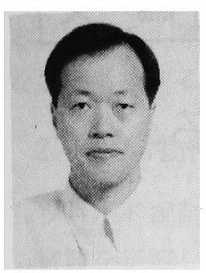
cer amic Engineering from YonSei University, Seoul, Korea, in 1980, and the M. S. in com puter science from New York Institute of Technology, New York, U. S. A., in 1987. He is now in the $\mathrm{Ph}$. D. program in information and computer science at Osaka City University. His research interests include genetic algorithms, machine learning, parallel processing, and artificial neural networks. He is a member of IEEE, ISAI.

Shoji Tatsumi (Non-member) He received the B.E., M.E.

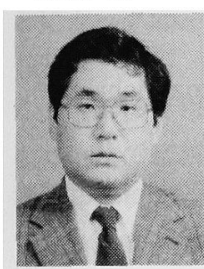
and Dr. Eng. degrees in Communication Engineering from Osaka University, in 1970, 72, and 78 , respectively. He is currently a Professor of information and computer science at Osaka City University. His current research interests are in the areas of genetic algorithm, statistical pattern recognition, decision. making problem, parallel processor for image processing, and VLSI-oriented connection network. He is a member of IEEE, JSAI, IPSJ, and JSSST.
Takaaki Okumoto (Member) He received the B. E., and $\mathrm{M}$

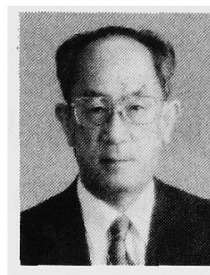

E. in Electrical Engineering from Kyoto University, in 1955, and 57, respectively. From 57 to 95 he had been at Osaka City University. He received Dr. Eng. degree from Kyoto University in 1972 . He is currently a Professor of information and computer science at Osaka Institute of Technology. His research inter. ests include learning recognition system, artificial intelligence, and modeling of heuristic behavior. He is a member of IEEE JSFTS, and IFSA 